\title{
Correlation of the EPR properties of perchlorotriphenylmethyl radicals and their efficiency as DNP polarizers
}

\author{
Debamalya Banerjee ${ }^{a}$, Juan Carlos Paniagua ${ }^{b}, V^{2}$ eronica Mugnaini ${ }^{c}$, Jaume Veciana $^{c}$, Akiva \\ Feintuch $^{\mathrm{a}}$, Miquel Pons ${ }^{\mathrm{d}, \mathrm{e}}$, Daniella Goldfarb ${ }^{\mathrm{a}, *}$ \\ ${ }^{\mathrm{a} D e p a r t m e n t}$ of Chemical Physics, Weizmann Institute of Science, Rehovot 76100, Israel; \\ bDepartament de Química Física \& Institut de Química Teòrica i Computacional (IQTC-UB), Universitat de \\ Barcelona. Martí i Franquès 1-11, 08028 Barcelona, Spain. \\ 'Institut de Ciència de Materials de Barcelona (ICMAB-CSIC), Bellaterra, Spain and Networking Research \\ Center on Bioengineering, Biomaterials, and NanoMedicine (CIBER-BBN), Bellaterra, Spain. \\ ${ }^{\mathrm{d}}$ Departament de Química Orgànica. Universitat de Barcelona. Martí i Franquès 1-11, 08028 Barcelona, \\ Spain. \\ e Institute for Research in Biomedicine (IRB Barcelona), Baldiri Reixac 10-12, 08028 Barcelona, Spain
}

\section{Abstract}

Water soluble perchlorinated trityl (PTM) radicals were found to be effective $95 \mathrm{GHz}$ DNP (dynamic nuclear polarization) polarizers in ex-situ (dissolution) ${ }^{13} \mathrm{C}$ DNP (Gabellieri et al, Angew Chem. Int. Ed. 2010, 49, 3360). The degree of the nuclear polarization obtained was reported to depend on the position of the chlorine substituents on the trityl skeleton. In addiiton, on the basis of the DNP frequency sweeps it was suggested that the ${ }^{13} \mathrm{C} N M R$ signal enhancement is mediated by the $\mathrm{Cl}$ nuclei. To understand the DNP mechanism of the PTM radicals we have explored the $95 \mathrm{GHz}$ EPR characteristics of these radicals that are relevant to their performance as DNP polarizers. The EPR spectra of the radicals revealed axially symmetric g-tensors. A comparison of the spectra with the ${ }^{13} \mathrm{C}$ DNP frequency sweeps showed that although the solid effect mechanism is operational the DNP frequency sweeps reveals some extra width suggesting that contributions from EPR forbidden transitions involving ${ }^{35,37} \mathrm{Cl}$ nuclear flips are likely. This was substantiated experimentally by ELDOR (electron-electron double resonance) detected NMR measurements, which map the EPR forbidden transitions, and ELDOR experiments that follow the depolarization of the electron spin upon irradiation of the forbidden EPR transitions. DFT (density functional theory) calculations helped to assign the observed transitions and provided the relevant spin Hamiltonian parameters. These results show that the ${ }^{35,37} \mathrm{Cl}$ hyperfine and nuclear quadrupolar interactions cause a considerable nuclear state mixing at $95 \mathrm{GHz}$ thus facilitating the polarizing of the $\mathrm{Cl}$ nuclei upon microwave irradiation. Overlap of $\mathrm{Cl}$ nuclear frequencies and the ${ }^{13} \mathrm{C}$ Larmor frequency further facilitates the polarization of the ${ }^{13} \mathrm{C}$ nuclei by 
spin diffusion. Calculation of the ${ }^{13} \mathrm{C}$ DNP frequency sweep based on the $\mathrm{Cl}$ nuclear polarization showed that it does lead to an increase in the width of the spectra, improving the agreement with the experimental sweeps, thus supporting the existence of the new heteronuclear assisted DNP mechanism.

\section{Introduction}

Dynamic nuclear polarization (DNP) is primarily used for enhancement of nuclear magnetic resonance signals in both the solid and the liquid states. This technique involves transfer of magnetization from pre-polarized electron spins to their neighboring nuclear $\operatorname{spin}^{1}$. The introduction of dissolution DNP by Golman and co-workers ${ }^{2}$ and low temperature magic angle spinning (MAS) DNP by Griffin and coworkers ${ }^{3}$, have demonstrated the applicability and potential of DNP to modern MRI and solid-state NMR and have revived the interest in the field. Moreover, recent developments in high power microwave sources have enabled considerable signal enhancements also in the liquid state at frequencies as high as $263 \mathrm{GHz}^{4}$.

Usually, DNP experiments are performed by dissolving radicals, commonly termed as the polarizing agents, in the host matrix containing the target nuclei. Then the electron spin thermal polarization is transferred to the nuclear spins by microwave $(\mathrm{mw})$ irradiation of the sample at a suitable frequency, mediated by the electron-nuclear dipolar interaction, resulting in the enhancement of the NMR signal ${ }^{1}$. In the liquid state the polarization transfer mechanism is dominated by the Overhauser effect ${ }^{5}$. In the solid state, if the width of the inhomogeneously broadened EPR lineshape of the polarizer is less than the corresponding nuclear Larmor frequency, $v_{n}$, the polarization transfer takes place via the anisotropic hyperfine interaction of an electron spin with a neighboring nuclear spin. This mechanism is known as the "solid effect" 6 . In a situation when the inhomogeneous EPR linewidth is larger than $v_{n}$, it is possible to locate two dipolar coupled electron spins separated in frequency by $v_{n}$. These electrons are capable of performing a flip-flop by releasing a quantum of energy matching $v_{n}$ which polarizes the nuclear spin. This mechanism is commonly referred to as the cross effect ${ }^{7}$. In the limit of high concentration of the polarizer, the mutual interactions among the electron spins are switched on. In such a system of many dipolar coupled electron spins, the polarization transfer takes place via a complex many body mechanism, commonly referred to as the "thermal mixing"8,9. In 
all of the above mechanisms the choice of the radical and its concentration are of outmost importance for achieving the best possible DNP enhancement.

Of particular interest is the dissolution (ex-situ) DNP ${ }^{2}$ where the NMR signal is observed at room temperature after a temperature jump of the sample polarized at very low temperature ( 1.5 K). A common polarizing agent for dissolution DNP applications involving the polarization of ${ }^{13} \mathrm{C}$ nuclei is the commercially available polarizing agent $0 X 63$, which has a triphenylmethyl skeleton (Fig. 1), and the transfer takes place through the solid effect DNP mechanism. Dissolution DNP is particularly advantageous within the context of hyperpolarized DNP-based in vivo NMR and MRI experiments where enhancements of the NMR signal by $>10,000$ times have been reported ${ }^{2}$. Examples of recent applications are in the field of $\mathrm{MRI}^{10}$, metabolomics ${ }^{11}$ and also, in obtaining heteronuclear multiple quantum coherence (HMQC) spectra of small molecules ${ }^{12,13}$.

Recently it has been demonstrated that water soluble perchlorinated trityl (PTM) radicals 1 and 2, shown in Fig. 1, can also be used to polarize ${ }^{13} \mathrm{C}$ nuclei in ex-situ DNP experiments with a rather good efficiency ${ }^{14,15}$. These radicals consist of a triphenylmethyl skeleton, as in OX63, but while in the 0X63 the central carbon atom is protected by six 5-membered rings containing sulphur (Fig. 1), in the PTMs the carbon bearing the unpaired electron is protected by six bulky chlorine atoms. In the PTM radicals it has been possible to substitute with carboxylic groups either the three para or the six meta positions with respect to the central carbon atom while all other positions on the phenyl rings are occupied by chlorine atoms.

The results obtained during earlier ex-situ DNP studies carried out with PTMs ${ }^{14,15}$, particularly the DNP frequency sweeps, suggested a chlorine-assisted polarization transfer mechanism for both radicals 1 and 2 . Radical 1 was reported to produce solution ${ }^{13} \mathrm{C}$ signal enhancement comparable to the commercial DNP polarizer OX63 whereas radical 2 gave a much lower DNP enhancement. In this latter case, the polarization was positive or negative depending on the polarized molecule, for reasons not yet very well understood. According to the proposed mechanism, the chlorine atoms present in the PTM radicals (Figure 1) act as core nuclei that are initially polarized by the electrons. Next, the ${ }^{13} \mathrm{C}$ nuclei present in the solvent molecules of the matrix are polarized via spin diffusion from the polarized chlorine nuclei. Moreover, it was suggested that strong mixing of the nuclear states by the ${ }^{35,37} \mathrm{Cl}$ nuclear quadrupole interaction could increase the transition probability of the forbidden EPR transitions enhancing the 
efficiency of solid effect DNP. Such an assumption was supported by Density Functional Theory (DFT) calculations that predicted large quadrupole coupling constants and non-negligible hyperfine couplings for the chlorine nuclei ${ }^{14}$. The proposed chlorine assisted polarization transfer could represent a new heteronuclear DNP enhancement mechanism with widespread possible applications in the field of both NMR and MRI.

To substantiate experimentally the above proposed hypothesis of chlorine assisted polarization transfer we have studied the EPR properties of PTM radicals $\mathbf{1}$ and $\mathbf{2}$ at W-band ( $95 \mathrm{GHz}$ ), the frequency at which the earlier ex-situ DNP work had been carried out. In particular, we have measured the frequencies of the forbidden transitions involving chlorine nuclei and calculated the expected transition probabilities. These were further used to compute the ${ }^{13} \mathrm{C}$ DNP frequency sweeps to account for their width and looking for the origin of the different polarization enhancement produced by the two PTM radicals.

The chlorine nuclear frequencies determined by ELDOR (electron-electron double resonance) detected $\mathrm{NMR}^{16}$, together with the DFT calculations, that gave the optimized structure for these radicals and their magnetic tensor parameters (Zeeman, hyperfine, quadrupole), provided support for the unique chlorine assisted polarization transfer mechanism of the perchlorinated trityl radicals under study.

\section{Methods}

Sample preparation: radicals 1 and $\mathbf{2}$ were synthesized as described earlier ${ }^{17,18}$. Solutions of 15 and $1 \mathrm{mM}$ radicals were prepared by dissolving radicals in the glass forming mixture DMSOwater mixture $(1: 1 \mathrm{v} / \mathrm{v})$. OX63 was purchased from GE Healthcare, AS and was dissolved in DSMO-water mixture $(1: 1 \mathrm{v} / \mathrm{v})$ to prepare $1 \mathrm{mM}$ solution.

Spectroscopic measurements: Unless otherwise specified, all the measurements were carried out at $40 \mathrm{~K}$ on a home built pulsed EPR spectrometer with a 2-channel mw bridge operating at a central frequency of $94.9 \mathrm{GHz}(\mathrm{W} \text {-band })^{19}$. Echo detected (ED) EPR spectra were recorded by the two pulse echo sequence $\{\pi / 2-\tau-\pi-\tau$-echo $\}$ with $t_{\pi}$ and $t_{x / 2}$ pulse durations of 30 and $60 \mathrm{~ns}$ respectively, and $\tau=800 \mathrm{~ns}$. Spin lattice relaxation times, $T_{1 e}$, were measured by a saturation recovery sequence $\left\{t_{s}-t-\pi / 2-\tau-\tau-\tau\right.$-echo $\}$ with a saturation pulse length of $t_{s}=10 \mathrm{~ms}$. ELDOR detected NMR spectra were recorded with the sequence shown in Fig. 4a. A high turning angle 
(HTA) pulse of $500 \mu \mathrm{s}$, applied at a frequency $v_{1}$, was used. The duration of the echo detection pulses were $t_{\pi / 2}=200 \mathrm{~ns}$ and $t_{n}=400 \mathrm{~ns}$ at a frequency $v_{0}=94.9 \mathrm{GHz}$. Spectra were recorded by measuring echo intensity as a function of $\Delta v=v_{1}-v_{0}$. For the ELDOR measurements, the frequency of the HTA pulse was fixed and the echo intensity was measured as a function of its length in the range of 0 to $135 \mathrm{~ms}$ in increments of $450 \mu \mathrm{s}$. The amplitude of the HTA pulse in the ELDOR measurements was determined by measuring the Rabi nutations at the respective frequency for different power attenuation settings.

DFT calculations: DFT calculations were carried out to optimize the geometry of radicals $\mathbf{1}$ and $\mathbf{2}$ and to obtain $\mathrm{g}$ matrices and hyperfine and quadrupole couplings of the $\mathrm{Cl}$ nuclei. All the calculations were performed with the program Orca version 2.7 revision $0^{20}$. The hybrid exchange-correlation functional B3LYP $\mathrm{P}^{21,22}$ was chosen together with the NMR/EPR-oriented basis set Iglo- $\mathrm{III}^{23}$, and all the electrons were included for every atom. Large numerical integration grids have been used to ensure rotational invariance: a Lebedev grid 6 (590 angular points, 40 radial points for $\mathrm{H}^{22} 45$ for second row atoms and 50 for $\mathrm{Cl}$ ) for SCF convergence, and a finer Lebedev grid 7 (770 angular points, 45 radial points for $\mathrm{H}, 46$ for second row atoms and 55 for $\mathrm{Cl}$ ) for an extra final iteration. The coupled-perturbed method was used for evaluating the spin-orbit coupling ${ }^{24}$.

Preliminary tests were performed for several chlorinated benzoic acids for which experimental quadrupole coupling constants were available $e^{25-27}$. As a general trend, calculated values showed an overestimation by about $5 \%$. These results did not improve when scalar relativistic corrections were included through the ZORA (zeroth-order regular approximation) or DKH (Douglas-Kroll-Hess) methods, which slightly increase the calculated values. In fact, the main source of error could well be the lack of solvent and temperature effects in the model, since quadrupolar couplings are usually about $5 \%$ higher in the gas phase than in solid state ${ }^{28,29}$. The COSMO (Conductor-Like Screening Model $^{30}$ ) polarizable continuum solvation model, available in the Orca program, proved to be insufficient to account for solvent effects, while the explicit inclusion of solvent molecules would greatly increase the computing time. On the other hand, quadrupole couplings slightly increase on going to very low temperatures ${ }^{29}$, which would partially compensate the solvent effect. Therefore, neither the solvent nor the scalar relativistic 
corrections were included in the final calculations. More computational details can be found in reference 14.

Spectral simulations: All the theoretical calculations were based on the following spin Hamiltonian:

$$
H=\beta \boldsymbol{\varpi} \bullet \cdot S-g_{n} B \cdot I+S \cdot \boldsymbol{D} I+I \cdot \boldsymbol{\nabla} I
$$

Where $\mathbf{S}$ and $I$ are the electron and the nuclear spin operators, $\mathbf{g}, \mathbf{A}$ and $\mathbf{P}$ are the Zeeman, hyperfine and the nuclear quadrupole tensors, $g_{n}$ is the isotropic nuclear Zeeman coupling constant and $\mathbf{B}$ is the external magnetic field. To simulate the ENDOR (electron-nuclear double resonance) spectra at different magnetic field positions within the EPR spectrum, the function 'salt' of EasySpin toolbox ${ }^{31}$ was used. The simulations were carried out for one equivalent unit (ring) of the radicals assuming identical results for all three units from symmetry. In the simulations the exact number of chlorine nuclei ( 4 for radical 1 and 3 for radical 2) of the radicals per equivalent units was considered. In the simulations both ${ }^{35} \mathrm{Cl}$ and ${ }^{37} \mathrm{Cl}$ were taken into account (natural abundance of $76 \%$ and $24 \%$ respectively) All possible orientations of the radical with respect to the external magnetic field were computed for a given position of the magnetic field.

$\mathrm{Cl}$ nuclear polarizations were calculated using a matlab code based on EasySpin functions ${ }^{31}$. The orientations $(\Omega)$ were given in terms of the Euler angles $(\alpha, \beta, \gamma$ in zyz convention with counter clockwise rotations) of the molecular g-axis system with respect to the external magnetic field $\mathbf{B}$. Calculations were carried out on a set of 1154 equally weighted Euler angles generated by ZCW algorithm ${ }^{32-34}$. Results were not improved further by use of a set of 3722 Euler angles (data not shown), i.e. the required convergence was achieved for 1154 orientations. The spin system operator corresponding to the irradiating $\mathrm{mw}$ field (for EPR transitions) or the irradiating $\mathrm{rf}$ field (for NMR transitions) were transformed to the diagonal frame of the spin Hamiltonian for each of these orientations. The probability of a specific EPR or NMR transition can be obtained from this transformed spin operator as a function of the molecular orientations. 


\section{Results}

$95 \mathrm{GHz}$ echo detected EPR spectra and spin lattice relaxation times

The W-band echo detected (ED) EPR spectra of $15 \mathrm{mM}$ solutions of radicals $\mathbf{1}$ and $\mathbf{2}$, recorded at $40 \mathrm{~K}$, (Fig. 2) exhibit powder patterns characteristic of an axially symmetric g-tensor. The spectra are quite similar except for a shift to higher g-values (lower field) for both $g_{\lrcorner}$and $g_{\|}$of radical 2. The g-values are listed in Table 1 along with the maximum width of the spectra. The same spectra were obtained for more diluted $(1 \mathrm{mM})$ solutions of the radicals. PTM radicals 1 and 2 show wider spectra than OX63 resulting from a considerably larger g-anisotropy (Fig. 2). An EPR spectrum of $0 \times 63$ recorded at $200 \mathrm{GHz}$ resolved a small axially symmetric g-anisotropy ${ }^{35}$. The larger anisotropy of the PTM radicals may be attributed to the spin-orbit coupling of the chlorine substituents.

The electron spin lattice relaxation times, $T_{1 e}$, at 10 and $40 \mathrm{~K}$, measured on $15 \mathrm{mM}$ samples at the position of maximum EPR intensity $\left(\mathrm{g}_{1}\right)$ by saturation recovery (see Fig. S2), are listed in Table 1. At a temperature of $40 \mathrm{~K}$ radical $\mathbf{1}$ exhibits a slightly shorter $\mathrm{T}_{1 \mathrm{e}}$ compared to radical $\mathbf{2}$. The difference between the $T_{1}$ values of radicals $\mathbf{1}$ and $\mathbf{2}$ increased significantly at $10 \mathrm{~K}$, being lower for radical 1.

The total EPR width ( $200 \mathrm{MHz} \mathrm{MHz}$ ) of radicals 1 and 2 largely exceed the width of the ${ }^{13} \mathrm{C}$ Larmor frequency $v_{13 \mathrm{c}} \sim 36.2 \mathrm{MHz}$ at $94.9 \mathrm{GHz}$ electron Larmor frequency), in contrast to the OX63 spectrum which exhibits the maximum total width of $60 \mathrm{MHz}$. Accordingly, the cross effect may be an efficient mechanism for the PTM radicals and it is not obvious that the solid effect mechanism, observed for OX63 under these conditions ${ }^{36}$, is the dominating DNP mechanism for the PTM radicals. Nonetheless, we exclude a dominating contribution of the cross-effect because the width of the frequency sweep exceeds significantly the width of the EPR spectrum. Considering the importance of the solid effect in OX63, we estimated the mw frequency dependence of the ${ }^{13} \mathrm{C}$ DNP enhancement that would result from the ED-EPR lineshape assuming solid effect mechanism (see Fig. S1, supplementary information for details). This estimated DNP sweep is shown in Fig. 3 (dashed line) and compared with the experimental DNP sweep measured at $1.5 \mathrm{~K}$ using $15 \mathrm{mM}$ of radicals 1 or 2 in neat pyruvic acid ${ }^{14,15}$. In this calculation we did not take into account any spin relaxation mechanism and ignored any 
orientation dependence of forbidden EPR transition probabilities. These are expected to affect the relative intensities in the DNP spectrum but not its maximum width. The general agreement between the calculated and the experimental spectrum, considering the very simplistic model used, is reasonable. There are, however, some noticeable important differences, such as the broader total width, particularly at the low frequency edge and the increased intensity and smearing of the lineshape in the high frequency region corresponding to $g_{\|}$in the DNP sweep. The differences in width can be partially accounted for by considering the effect of EPR forbidden transitions involving ${ }^{35,37} \mathrm{Cl}$ flips (see below). We note that a similar calculation of the OX63 ${ }^{13} \mathrm{C}$ DNP sweep from the OX63 EPR spectrum gave a very good agreement with the experimental DNP frequency sweep (not shown).

Table 1: g-values, $T_{1 e}$ and EPR spectral width, $\Delta v$, of the PTM radicals.

\begin{tabular}{|l|l|l|l|l|l|l|}
\hline Radical & $\begin{array}{l}\text { Sample } \\
\text { Concentration }\end{array}$ & $\mathrm{g}_{\lrcorner}$ & $\mathrm{g}_{\mathrm{I}}$ & $\Delta v(\mathrm{MHz})$ & $\begin{array}{l}\mathrm{T}_{1 \mathrm{e}}, 40 \mathrm{~K} \\
(\mathrm{~ms})\end{array}$ & $\begin{array}{l}\mathrm{T}_{1 \mathrm{e}}, 10 \mathrm{~K} \\
(\mathrm{~ms})\end{array}$ \\
\hline $\mathbf{1}$ & $15 \mathrm{mM}$ & 2.00005 & 2.00271 & 186 & 9.6 & 183.4 \\
\hline $\mathbf{2}$ & $15 \mathrm{mM}$ & 2.00121 & 2.00415 & 215 & 11.0 & 242.4 \\
\hline
\end{tabular}

\section{Mapping the forbidden EPR transitions}

At the core of the DNP solid effect lays the excitation of forbidden EPR transitions, which lead to nuclear polarization. Accordingly, we have used ELDOR-detected NMR to map these transitions for radicals $\mathbf{1}$ and $\mathbf{2}$. In these spectra the frequency axis $\left(\Delta v=v_{1}-v_{0}\right)$ corresponds to the nuclear frequencies ${ }^{37}$.

The ELDOR detected NMR spectra measured at different magnetic fields within the inhomogeneous EPR spectra of radicals 1 and 2 are presented in Fig. 5 (top). Due to the high selectivity of the pulses and the g-anisotropy, at each selected field only a subset of molecules with specific orientations relative to the external magnetic field are excited and contribute to the ELDOR detected NMR spectrum. Such a series of spectra is usually referred to as orientation selective spectra ${ }^{37}$ This set of measurements has been carried out on $1 \mathrm{mM}$ samples of the 
radicals since at a concentration of $15 \mathrm{mM}$ the ELDOR detected NMR are broadened due to the shortening of the phase memory time because of excessive spin-spin interactions (data not shown). The fields at which the measurements were performed are marked on the ED-EPR spectrum of radical 1 (Fig. 2) and similar relative positions were used for radical $\mathbf{2}$. The series of spectra of the two radicals are rather similar; both sets exhibit only a minor dependence on the selected field position. This indicates a weak orientation selection probably due to the symmetry of the radicals and the large number of chlorines. The spectra have three distinct features: (1) a broad band at $\sim 25 \mathrm{MHz}$, (2) a very broad transition between 30-45 MHz and (3) a relatively sharp transition around $65 \mathrm{MHz}$. Measurements on PTM radical derivatives, similar to 1 and $\mathbf{2}$, where the $\mathrm{Na}^{+}$counterions were replaced by protons (i.e., the radical carboxylic acid derivatives) gave identical results, whereas measurements on OX63 show only the central self ELDOR peak at $\Delta v=0$ (Figure S3). Accordingly, we assign the signals observed for radicals $\mathbf{1}$ and $\mathbf{2}$ to forbidden EPR transitions associated with flips of ${ }^{35,37} \mathrm{Cl}$ nuclei.

DFT calculations predict for radicals $\mathbf{1}$ and $\mathbf{2}$ a large ${ }^{35,37} \mathrm{Cl}$ quadruple coupling constant that dominates the nuclear Hamiltonian terms, in agreement with previous measurements of the nuclear quadrupole coupling on aromatic chlorinated molecules ${ }^{29}$. To illustrate the transitions involved in such a system we present in Fig. $4 \mathrm{~b}$ a schematic energy level diagram of an electron coupled to a nucleus of $I=3 / 2$ with a nuclear quadrupole coupling constant $e^{2} q|Q| /(4 \mid(2 \mid-1) h)$ comparable to the nuclear Zeeman or the corresponding hyperfine coupling. This large quadrupolar coupling results in orientation dependent mixing of the nuclear Zeeman based states and the levels cannot be easily associated to nuclear quantum numbers. In Figure $4 \mathrm{~b}$, 'forbidden' $\left(\Delta \mathrm{M}_{\mathrm{S}}= \pm 1, \Delta \mathrm{M}_{1} \neq 0\right)$ and 'allowed' $\left(\Delta \mathrm{M}_{\mathrm{S}}= \pm 1, \Delta \mathrm{M}_{1}=0\right)$ EPR transitions have been arbitrarily assigned to pairs of energy levels and the effect of the saturation of one particular forbidden transition (3-5) on the populations is presented.

\section{DFT calculations and simulation of the ENDOR spectra of radicals $\mathbf{1}$ and $\mathbf{2}$}

The spin Hamiltonian parameters obtained by DFT calculations for each of the chlorine nuclei types in radicals 1 and $\mathbf{2}$ are listed in Table 2 for ${ }^{35} \mathrm{Cl}$. The ELDOR detected NMR spectra depend on a rather large set of spin Hamiltonian parameters. These include the hyperfine and quadrupole tensors of each type of chlorine atoms, amounting to 11 per chlorine (Table 2). We have not attempted to extract them from fitting the orientation selection series of spectra. 
Rather we have used parameters obtained from DFT calculations to simulate spectra which were then compared with the experimental ones. A series of simulated ENDOR (electronnuclear double resonance) spectra of the two radicals at each of the experimentally chosen magnetic fields within the EPR spectra are shown in Fig. 5. The spectra contain contributions from both ${ }^{35} \mathrm{Cl}$ and ${ }^{37} \mathrm{Cl}$ according to their relative natural abundances. The spin Hamiltonian parameters for ${ }^{37} \mathrm{Cl}$ were obtained from the values of Table 2 after appropriate scaling determined by the relative nuclear gyromagnetic ratio $(\gamma)$ and quadrupole moment $(Q) .{ }^{38}$ ELDOR detected NMR and ENDOR spectra exhibit the same nuclear frequencies. However, the relative intensities in the spectra may be different as the former depends on the probability of the forbidden electron spin transitions whereas the latter depends on the probabilities of the allowed NMR transitions. Therefore, the comparison of ENDOR simulations and experimental ELDOR detected NMR spectra is restricted to the frequencies. . As shown in Fig. 5 (bottom), the simulations reproduced rather well all the main features of the ELDOR detected-NMR spectra. The good agreement between the experimental and calculated spectra provides a validation of the DFT calculated parameters and confirms the assignment of the signals to chlorine.

Table 2: Principal values and orientations with respect to the g-tensor for hyperfine and the quadrupole tensors obtained by DFT calculations and subsequently used for the simulation of the ENDOR spectrum of radicals 1 and 2 . All values refer to ${ }^{35} \mathrm{Cl}$. The ${ }^{37} \mathrm{Cl}$ values can be obtained after appropriate scaling determined by the relative nuclear gyromagnetic ratio $(\gamma)$ and quadrupole moment $(Q)^{38}$.

\begin{tabular}{|c|c|c|c|c|c|c|c|c|}
\hline Radical & $\begin{array}{l}\text { Chlorine } \\
\text { Position }\end{array}$ & $\begin{array}{l}A_{x x} \\
(M H z)\end{array}$ & $\begin{array}{l}A_{y y} \\
(M H z)\end{array}$ & $\begin{array}{l}\mathrm{A}_{\mathrm{zz}} \\
(\mathrm{MHz})\end{array}$ & $\begin{array}{l}\text { Euler } \\
\text { Angles }^{*} \\
\alpha \beta \gamma\end{array}$ & $\begin{array}{l}\mathrm{e}^{2} \mathrm{qQ} / \mathrm{h} \\
(\mathrm{MHz})\end{array}$ & $\eta$ & $\begin{array}{l}\text { Euler } \\
\text { Angles }^{*} \\
\alpha \beta \gamma\end{array}$ \\
\hline \multirow[t]{2}{*}{1} & ortho & -0.17 & -2.07 & 2.50 & $\begin{array}{l}7.0 \\
45.2 \\
36.8\end{array}$ & -76.33 & 0.153 & $\begin{array}{l}-59.9 \\
136.3 \\
41.7\end{array}$ \\
\hline & meta & 0.42 & -1.53 & 1.62 & $\begin{array}{l}43.0 \\
71.36 \\
116.8\end{array}$ & -76.20 & 0.138 & $\begin{array}{l}59.5 \\
130.5 \\
42.8\end{array}$ \\
\hline 2 & ortho & 0.16 & -1.76 & 2.56 & 177.4 & -74.40 & 0.114 & 61.8 \\
\hline
\end{tabular}




\begin{tabular}{|l|l|l|l|l|l|l|l|l|}
\hline & \multirow{2}{*}{} & & & & $\begin{array}{l}50.1 \\
45.7\end{array}$ & & & \\
\hline & para & -0.72 & -1.64 & 3.27 & 93.4 & -71.00 & 0.111 & 42.05 \\
& & & & & 56.8 & & & 90.0 \\
& & & & & 0 & & & -138.4 \\
\hline
\end{tabular}

* Orientation with respect to the g-tensor of the molecule in zyz convention with counter clockwise rotations.

One notable aspect of all the ELDOR- detected NMR spectra is the broad feature in the frequency range of $30-45 \mathrm{MHz}$ which overlaps the ${ }^{13} \mathrm{C}$ Larmor frequency, $v_{13 c}$. The frequency match between some of the forbidden transitions involving chlorine nuclei and $v_{13 c}$ at the magnetic field used in the DNP experiments can provide a mechanism for spin diffusion from the initially polarized ${ }^{35,37} \mathrm{Cl}$ nuclei to bulk ${ }^{13} \mathrm{C}$ nuclei, as originally suggested ${ }^{14,15}$. On the other hand, the broad spread of forbidden transitions originating from the quadrupolar nature of chlorine may allow efficient DNP to ${ }^{35,37} \mathrm{Cl}$, and indirectly to ${ }^{13} \mathrm{C}$, at a broad range of $\mathrm{mw}$ frequencies. We note that the overlap of the ${ }^{35,37} \mathrm{Cl}$ nuclear transitions with the ${ }^{13} \mathrm{C}$ larmor frequency depends on the spectrometer frequency. The overlap observed at $95 \mathrm{GHz}$ improves significantly at $140 \mathrm{GHz}$, while at $270 \mathrm{GHz}$ there is no overlap (see Fig. S4).

To verify the effectiveness of the $m w$ irradiation at $v_{13 c}$, we have set the frequency of the HTA pulse to $v_{13 c}$ and followed the echo intensity as a function of irradiation time and power. Such an experiment provides the electron spin depolarization rate, which is the initial step of DNP ${ }^{39}$. The results, obtained at a magnetic field corresponding to the maximum EPR intensity for radicals $\mathbf{1}$ and $\mathbf{2}$ are given in Figure 6a-d. The echo intensity drops drastically within an irradiation time up to $t_{\text {HTA }} \sim 2 \mathrm{~ms}$. With longer irradiation, the echo intensity levels off to a constant value for higher mw power (Figure 6a) or recovers marginally from the minimum value for lower mw power (Figure 6d). The steady state value decreases with increasing mw power. The initial decay in the echo intensity characterizes the loss of electron polarization at the given irradiation frequency. The percentage of polarization loss is always greater in radical $\mathbf{1}$, which is a better DNP polarizer ${ }^{14,15}$, than in radical 2 independent on the mw power. 


\section{Calculations of the nuclear polarization and the associated forbidden electronic transitions}

In an attempt to account for the width of the ${ }^{13} \mathrm{C}$ DNP frequency sweep and to identify the source of the differences in the DNP performance of the two radicals in ex-situ DNP ${ }^{14,15}$, we computed the nuclear polarizations of each of the ${ }^{35} \mathrm{Cl}$ and ${ }^{37} \mathrm{Cl}$ nuclei associated with particular forbidden EPR transitions. This was done by calculating the frequencies and transition probabilities of the forbidden EPR transitions using the hyperfine and quadrupole tensors obtained from the DFT calculations (see Table 2). We assumed that each chlorine nucleus interacts with the electron spin independently, to avoid calculations of a complicated, multi nuclear spin Hamiltonian that leads to many possible transitions involving multi-nuclear flips with small transition probabilities. For a nuclear spin $\mathrm{I}=1 / 2$, where there are only two NMR transitions, the saturation of any of the two EPR forbidden transitions will result in the two NMR transitions having the same polarization, either positive or negative. In contrast, for $1>1 / 2$ nuclei the situation is more complex as several NMR transitions are affected by the saturation of a particular forbidden EPR transition and these may have different polarizations. For example, let us consider the forbidden 5-3 EPR transition highlighted in Fig. 4b. Saturation of this transition will increase the population of level 3 and diminish the populations from level 5 . This will result in a positive polarization for the 3-2 and 3-1 NMR transitions and a negative polarization for the 4-3 transition. These transitions belong to the upper $M_{s}=1 / 2$ manifold. In the lower $M_{s}=-1 / 2$ manifold all the three affected NMR transitions 5-6, 5-7 and 5-8, have positive polarizations. Similarly, for each orientation of the magnetic field (denoted by $\Omega$ in this text), one can map all the forbidden EPR transitions and the frequency and polarization of all associated NMR transitions.

To put this argument into a quantitative framework we assume that the mw irradiation leads to partial saturation of populations of levels $j$ and $k$ separated by frequency $v_{j, k}{ }^{e}$. If $p^{0}(j)$ and $p^{0}(k)$, and $p(j)$ and $p(k)$ are the populations of levels $j$ and $k$ before and after the $m w$ irradiation, respectively, the population difference of levels $j$ and $k$ is:

$$
p(k)-p(j)=\frac{p^{0}(k)-p^{0}(j)}{1+W_{j, k}^{e} S}
$$

and 
$p(k)+p(j)=p^{0}(k)+p^{0}(j)$

Equation 2a follows from the steady state solution of the Bloch equation ${ }^{6}$ and Eq. $2 b$ from the conservation of the total population of levels $\mathrm{k}, \mathrm{j}$. The latter assumption means that we ignore any redistribution of populations between the multi-level system due to electron and nuclear spin lattice relaxations and electron-nuclear cross relaxation. In Eq. $2 a, W_{j k}^{e}$ is the probability of the k-j EPR transition and it was obtained from the spin Hamiltonian in Eq.1, the DFT parameters listed in Table 2 and the experimentally determined g-values. $S$ is the saturation factor, given by, $S=\left(\gamma B_{1}\right)^{2} T_{1} T_{2}$, where $B_{1}$ is the irradiating microwave field, and $T_{1}$ and $T_{2}$ are the spin-lattice and spin-spin relaxation rates of the $k, j$ transition, respectively. Using Eqn. $2 a$ and $2 b$ we obtain:

$$
\begin{aligned}
& p(j)=\frac{W_{j, k}^{e} S\left(p^{0}(k)+p^{0}(j)\right)+2 p^{0}(j)}{2\left(1+W_{j, k}^{e} S\right)} \\
& p(k)=\frac{W_{j, k}^{e} S\left(p^{0}(k)+p^{0}(j)\right)+2 p^{0}(k)}{2\left(1+W_{j, k}^{e} S\right)}
\end{aligned}
$$

The enhancement ${ }^{C l, N} \varepsilon_{l, j}$ of a particular NMR transition i-j of a chlorine type $\mathrm{N}$ for a given molecular orientation, corresponding to an EPR forbidden transition j-k with frequency $v_{j, k}{ }^{e}$ is:

$$
{ }^{C l, N} \varepsilon_{i, j}\left(v_{j, k}^{e}, \Omega, S\right)=\frac{p^{0}(i)-p(j)}{p^{0}(i)-p^{0}(j)}
$$

Note that $p^{0}(i)=p(i)$. Next we assume that all polarized chlorine nuclei that have resonance frequencies $v_{i, j}^{n}$ within the range $v_{13 c} \pm \Delta$ will transfer their polarization, weighted by the corresponding NMR transition probabilities $W_{i, j}{ }^{n}$, to the ${ }^{13} \mathrm{C}$ nuclei in the bulk by spin diffusion while keeping the sign of their polarization.

After mapping all the NMR transitions with frequencies in this range, we computed the expected polarization enhancement of the chlorine nuclei as a function of the frequency of the microwave irradiation $v_{\mathrm{mw}}$ that can affect the forbidden EPR transitions related to those NMR transitions. This in turn gave the estimated ${ }^{13} \mathrm{C}$ enhancement, ${ }^{\mathrm{C}, \mathrm{N}} \varepsilon\left(v_{\mathrm{mw}}, \mathrm{S}, \Omega\right)$, arising from a particular chlorine nucleus, $N$. The enhancements ${ }^{C, N} \varepsilon\left(v_{m w}, S, \Omega\right)$ were separated into positive and negative values, ${ }^{\mathrm{C}, \mathrm{N}} \varepsilon\left(\nu_{\mathrm{mw}}, \mathrm{S}, \Omega, \pm\right)$. 
The ${ }^{\mathrm{C}, \mathrm{N}} \varepsilon\left(v_{\mathrm{mw}}, \mathrm{S}, \pm\right){ }^{13} \mathrm{C}$ bulk polarizations were calculated by summing it over all $\mathrm{Cl} \mathrm{NMR}$ transitions with weights given by $W_{i, j}{ }^{n}$, within the $v_{m w} \pm \delta$ range and integrating over all possible values of the orientation $\Omega$. This was done separately for the positively and negatively polarized $\mathrm{Cl} N \mathrm{NR}$ transitions:

${ }^{C, N} \varepsilon\left(v_{m w}, S, \pm\right)=\int\left[\sum_{k, j} \sum_{i}^{C l, N} \varepsilon_{i, j}\left(v_{j, k}^{e}, \Omega, S, \pm\right) W_{i, j}^{n} \Re\left(v_{m w}-v_{j, k}^{e}\right) \Re\left(v_{13 C}-v_{i, j}^{n}\right)\right] d \Omega$

where $\Omega$ and $\mathrm{d} \Omega$ are expressed through the Euler angles that give the orientation of the magnetic field within the molecular $g$ frame.

The function $\Re$ is a step function defined as:

$\mathfrak{R}(x)=1$ if $-\mathrm{R} \leq \mathrm{x} \leq \mathrm{R}$

$\mathfrak{R}(x)=0$ elsewhere

$R=\delta$ for the EPR transitions and $R=\Delta$ for the NMR transitions. $\delta$ and $\Delta$ act as effective linewidths. This means that microwave irradiation at $v_{\mathrm{mw}}$ affects all the EPR forbidden transitions having frequencies in the range $v_{\mathrm{mw}} \pm \delta$ and a certain NMR transition will be taken into consideration only if its frequency lies in the range $v_{13 c} \pm \Delta$. In practice $\delta=0.5 \mathrm{MHz}$ has been used for all the calculations. Separation of the enhancements ${ }^{\mathrm{C}, \mathrm{N}} \varepsilon\left(v_{\mathrm{mw}}, \mathrm{S}, \Omega\right)$ in positive and negative values does not introduce a significant numerical error in the calculations (Fig. S6 of SI) and it does show the presence of enhancements of both sign for a large range of microwave frequencies.

Due to the discrete nature of the calculation, smoothing and averaging over these data sets were required. At first, more data points were created by numerical interpolation. Then, a builtin matlab function was used to smooth the data by computing a moving average over 40 points. We also carried out this interpolation and smoothing on the complete data set without separating it into positive and negative polarization and the differences obtained where subtle (see Fig S6).

After that, the positive and negative enhancements were re-combined (see Fig. S5 of SI).

${ }^{C, N} \varepsilon\left(v_{m w}, S\right)={ }^{C, N} \varepsilon\left(v_{m w}, S,+\right)+{ }^{C, N} \varepsilon\left(v_{m w}, S,-\right)$ 
Equation 6 represents the numerically constructed ${ }^{13} \mathrm{C}$ DNP frequency sweep for a particular chlorine type $N$. At the end, a FFT smoothing filter in Origin 8.1 (OriginLab ${ }^{\circledR}$ ) was used to generate the final dataset. In the above calculation, the effect of relaxation (both electronic and nuclear) and microwave power on the polarization were included through the phenomenological saturation factor $S$. The saturation limit enhancement value, ${ }^{C, N} \varepsilon\left(v_{m w}\right)$ was obtained for $S \rightarrow \infty$, where the populations of levels $j, k$ become equal:

$\lim _{S \rightarrow \infty} p(j)=p(k)=\frac{\left(p^{0}(k)+p^{0}(j)\right)}{2}$

The results of the theoretical calculations for both the PTM radicals are shown in Figs. 7, 8 and 3. The dependence of ${ }^{\mathrm{C}, \mathrm{N}} \varepsilon\left(v_{\mathrm{mw}}, \mathrm{S}\right)$ for the ortho ${ }^{35} \mathrm{Cl}$ of radical 1 on the saturation factor is demonstrated in Fig. 7 alongside its absolute value. The DNP frequency sweeps gradually approach the saturation limit curve (i.e. equal populations in the energy levels) for higher values of the saturation factor $S$. Next, the calculation was performed in the saturation limit with two different values of the $\mathrm{Cl}^{13} \mathrm{C}$ polarization transfer bandwidth $(\Delta)$, namely $0.5 \mathrm{MHz}$ and $1 \mathrm{MHz}$, for all different $\mathrm{Cl}$ substituents. As it is evident from Figs. 8, there is not much difference between the results obtained using two values of $\Delta$, and therefore $\Delta=1 \mathrm{MHz}$ has been used throughout the calculations.

In an effort to construct the theoretical analogue of the frequency sweep DNP data for each radical, we considered the saturation limit data obtained with $\Delta=1 \mathrm{MHz}$ and performed the sum over the enhancement values obtained from different $\mathrm{Cl}$ substituents and the $\mathrm{Cl}$ isotopes.

${ }^{C} \varepsilon\left(v_{m w}\right)=\sum_{L} n_{L}{ }^{C, N} \varepsilon\left(v_{m w}\right)$

In eq. $8, n_{L}$ corresponds to the number of the different $\mathrm{Cl}$ nuclei in the PTM radicals. As there are 6 ortho $\mathrm{Cl}$ and 6 meta $\mathrm{Cl}$ in radical 1 and 6 ortho $\mathrm{Cl}$ and 3 para $\mathrm{Cl}$ in radical $2, \mathrm{n}^{1}$ ortho $=6, n_{\text {meta }}^{1}=6$ and $\mathrm{n}^{2}$ ortho $=6, n_{\text {para }}^{2}=3$ were used. In these calculations we considered contributions from both ${ }^{35} \mathrm{Cl}$ and ${ }^{37} \mathrm{Cl}$ according to their natural abundance $\left(\sim 7 \%\right.$ of ${ }^{35} \mathrm{Cl}$ and $\sim 24 \%$ of $\left.{ }^{37} \mathrm{Cl}\right)$ as described earlier. The results are shown in Fig. 3 (solid line) after renormalization to fit the experimental results and in Fig. S7. The latter predicts a better enhancement by about $20 \%$ for radical 1. 
Comparison of the calculated frequency sweeps with the experimental ones and those derived from the EPR powder pattern considering direct transfer of the electron polarization to bulk ${ }^{13} \mathrm{C}$ for radical $\mathbf{1}$ and $\mathbf{2}$ is presented in Fig. 3. It shows that the calculation based on the $\mathrm{Cl}$ assisted polarization transfer accounts better than the standard solid effect for the total width of the spectrum, particularly at the low frequency end, although the peak positions is still a Ittle too high. The relative intensities in the high field end do not agree well . This will be discussed further in the next section.

\section{Discussion}

The spectral widths of the PTM radicals, due to g-anisotropy, are about three times larger than that of OX63 (Fig. 1). Accordingly, for a given mw irradiation, at least three fold less electron spins are affected by the mw irradiation as compared to OX63. Nonetheless, the efficiency of radical 1 as a polarizing agent in ex-situ DNP was reported to be close to that of $0 \times 63^{15}$. This indicates a higher DNP enhancement per excited spins for radical 1 that can be explained partially by contributions from an efficient $\mathrm{Cl}$ assisted polarization transfer mechanism.

Although the nuclei closest to the unpaired electron, termed core nuclei, have the largest hyperfine interactions and therefore they get easily polarized because of the larger transition probability of the corresponding forbidden transitions, their polarization is transported very inefficiently by spin diffusion to the bulk nuclei due to frequency mismatch ${ }^{40}$. This frequency mismatch cannot be avoided in the homonuclear case but it is possible in a heteronuclear case. In the case of the PTM radicals the ELDOR detected NMR measurements showed that there is a considerable overlap of the frequencies of the $\mathrm{Cl}$ core nuclei with the ${ }^{13} \mathrm{C}$ Larmor frequency at 95 $\mathrm{GHz}$. This overlap opens an effective channel for heteronuclear spin diffusion between the $\mathrm{Cl}$ core nuclei and nearby ${ }^{13} \mathrm{C}$ nuclei. This process can in principle be both inter- and intramolecular. Considering the abundance of receiving ${ }^{13} \mathrm{C}$ nuclei in enriched substrates or in the presence of carbon bearing solvents, transfer of polarization from the core $\mathrm{Cl}$ nuclei is most probably inter-molecular. Next, homonuclear ${ }^{13} \mathrm{C}$ spin diffusion transfers the polarization to the rest of the ${ }^{13} \mathrm{C}$ in the sample (solute and solvent).

The ex-situ DNP experiments performed ${ }^{14,15}$ with PTM radicals showed that radical $\mathbf{1}$ is a more effective polarizing agent than radical 2 for the same solute and solvent. The ELDOR data 
presented in this work indeed indicate that the polarization of the $\mathrm{Cl}$ core nuclei is more effective for radical 1, consistent with the above observation. As illustrated in Fig. 6, upon irradiation of a EPR forbidden transition associated with a $\mathrm{Cl}$ nuclear frequency equal to $v_{13 \mathrm{C}}$, radical 1 loses more electron polarization than radical 2 ( 90\% and 50\% for radical 1 and 2 at highest mw power used, respectively). The magnitude of the difference in the ex-situ DNP enhancement of radical $\mathbf{1}$ and $\mathbf{2}$ is however much larger than that observed in the ELDOR experiments. Whereas the ex-situ DNP enhancements by radical 1 is at least $\sim 3$ times ${ }^{15}$ (for ${ }^{13} \mathrm{C}$ labeled urea) than radical 2 , the electron polarization loss of the same radical at $v_{13 c}$ is not even twice as strong; even for the highest $\mathrm{mw}$ power used $\left(\omega_{1} / 2 \pi \sim 12 \mathrm{MHz}\right.$, figure $\left.6 \mathrm{a}\right)$. This indicates that additional factors affect the final ex-situ DNP enhancement. Differences in electron and spin lattice nuclear relaxation times could also contribute to the differences ${ }^{40}$. Recently, it has been reported that addition of small amount of $\mathrm{Gd}^{3+}$ ion affects ${ }^{13} \mathrm{C}$-DNP favorably and this was attributed to the shortening the $T_{1 e}$ relaxation of the OX63 polarizing agent ${ }^{36}$. Our $T_{1 e}$ results, although not at $1.5 \mathrm{~K}$, show that $\mathrm{T}_{1 \mathrm{e}}$ of radical 1 is shorter at both 40 and $10 \mathrm{~K}$.

Once the spectral overlap of the ${ }^{35,37} \mathrm{Cl}$ and ${ }^{13} \mathrm{C}$ nuclear frequencies has been established experimentally and the spin Hamiltonian parameters have been determined by DFT and shown to reasonably reproduce the observed nuclear frequencies, it was possible to proceed and use these parameters to calculate the frequencies and transition probabilities of forbidden EPR transitions and the associated NMR transitions. From this, the polarization of the associated NMR transitions and, eventually, the ${ }^{13} \mathrm{C}$ DNP frequency sweeps were obtained. An important aspect of this calculation is that the high nuclear spin leads to the presence of nuclear polarization enhancements of both signs for the entire range of forbidden electron spin transition frequencies. In such cases, the presence of more than one nuclear transition in each $\mathrm{M}_{\mathrm{s}}$ manifold leads to both positive and negative nuclear enhancements for a particular forbidden EPR transition (or microwave irradiation) frequency (Fig. S5). This is in contrast to $I=1 / 2$ spin systems where the sign of the nuclear enhancement is determined by the microwave irradiation frequency relative to the allowed transition.

${ }^{13} \mathrm{C}$ DNP frequency sweep are considerably wider than the EPR spectra of the radicals indicating that the contributions of the solid effect mechanism is most significant. However, ${ }^{13} \mathrm{C}$ DNP sweeps reconstructed from the ED-EPR spectra assuming the solid effect mechanism and direct 
polarization transfer to ${ }^{13} \mathrm{C}$ did not reproduce all features of the experimental spectra. Most prominent is the mismatch in the spectral width, particularly in the low frequency side. The calculations based on the ${ }^{35,37} \mathrm{Cl}$ assisted polarization transfer, displayed in Fig. 3, exhibit a better agreement with the experiment in terms of the total with of the DNP sweeps, particularly at the low frequency edge. This calculation was based on the polarization enhancement of $\mathrm{Cl}$ nuclei with frequencies in the vicinity of $v_{13 c}$. This finding supports the idea of the chlorine assisted heteronuclear polarization transfer to ${ }^{13} \mathrm{C}$ discussed earlier. However, some width is still missing and the intensities in the high frequency end also do not match. Next we discuss elements that were not taken into account in the calculations of the ${ }^{13} \mathrm{C}$ DNP sweeps and could account for this mismatch.

The theoretical DNP sweeps for both PTM radicals, as shown in Fig. 3, were obtained as sum of the data for different chlorine substituents at the saturation-limit $(S \rightarrow \infty)$ according to their numbers in the PTM radicals. The simulations of the DNP frequency sweeps were highly simplified and were based only on the spin Hamiltonian parameters derived from the DFT calculations while the relaxation was introduced only through the saturation factor. The nuclear and electron spin lattice and cross relaxation rates, which are major factors in the determination of the efficiency of the DNP process and might affect the shape of the frequency sweep ${ }^{40}$, were not taken into account. As evident from Fig. 7, a high value of the saturation factor increases the contributions from forbidden EPR transitions that contribute to the higher frequency range of the calculated DNP frequency sweep and have low transition probabilities. This improves the agreement with the experimental DNP data.

We also ignored any possibility of direct polarization transfer from the electron spin to ${ }^{13} \mathrm{C}$ nuclei. This is expected to affect the DNP lineshape but not increase its maximal width and therefore it will not account for width missing at the low frequency edge. Moreover, considering the EPR linewidth we cannot exclude the possibility that the cross-effect mechanism has also a significant contribution. Such a contribution, that could also occur via the $\mathrm{Cl}$ nuclei, will not contribute intensities beyond the width of the EPR spectrum and therefore will also not account for the missing width. These two can account for the intensity differences in the high frequency end. As we have shown that the extra width of DNP is determined by the spin Hamiltonian parameters and the solid effect mechanism we tested the effect of a $\pm 10 \%$ 
variation in the hyperfine or quadrupolar parameters on the calculated DNP frequency sweep. This was carried out for the ortho ${ }^{35} \mathrm{Cl}$ of radical 1 and the results (see Fig. S8) show that while for the hyperfine interaction the effect is insignificant, a $10 \%$ change in the quadrupole coupling constant may have a significant effect on the width. Accordingly, the mismatch observed between the calculated DNP sweeps and the experimental DNP sweeps may be due a small variation between the DFT predicted quadrupolar parameters and the real ones. To find the parameters through fitting is, however, impractical because it involves too many parameters as two types of $\mathrm{Cl}$ have to be taken into account

The calculations also yield higher enhancement for radical 1 that stems both from the more efficient polarization of its ortho $\mathrm{Cl}$ due to more favorable spin Hamiltonian parameters and due to the larger number of $\mathrm{Cl}$ nuclei (see Fig. S7 in SI). The difference, $20 \%$, however is much smaller than observed in the ex-siue experiments but similar to that found in the ELDOR measurements (Fig. 6) In these calculations we assumed a single $S$ value for all the transitions. But in principle, $S$ is transition dependent through relaxation. In addition, the step of the $\mathrm{Cl}$ polarization transfer to the ${ }^{13} \mathrm{C}$ was not taken into account explicitly but rather through empirical factors such as $\Delta$ and the number of different types of $\mathrm{Cl}$ atoms $n_{L}$.

The stage involving the heteronuclear $\mathrm{Cl}^{13} \mathrm{C}$ polarization transfer may be different in the two radicals due to their different structures and number of chlorine substituents, 12 in radical 1 and 9 in radical 2. Steric effects may favor the approach of solute molecules to specific chlorine nuclei and therefore, alternative transfer pathways may be available depending on the nature of the radical and the nearest molecules in the glass. The calculation of DNP frequency sweeps assume that different chlorine substituents play equal roles in the heteronuclear assisted polarization transfer process. But in reality the efficiency of the $\mathrm{Cl}^{13}{ }^{13} \mathrm{C}$ transfer for different types of $\mathrm{Cl}$ atoms (in terms of the positions in the PTM radicals) is not really known and it can be different due to steric and chemical factors mentioned above.

In this work, we focused on the experimental and calculated EPR characteristics of the PTM radicals and explored their relation to the radicals' DNP efficiencies. In addition we substantiated experimentally the hypothesis of a heteronuclear assisted polarization transfer mechanism for ${ }^{13} \mathrm{C}$ suggested earlier ${ }^{14,15}$. However, to fully comprehend the DNP mechanism of these new radicals and the source of the difference between radicals $\mathbf{1}$ and $\mathbf{2}$ future systematic 
studies of the solid state DNP stage should be carried out. These should explore quantitatively the dependence of the ${ }^{13} \mathrm{C}$ enhancement obtained with both radicals on various experimental parameters, such as solvent character, temperature and particularly microwave power. The calculations predict a strong dependence of the lineshape on the microwave power and this can be verified experimentally.

\section{Summary and Conclusions}

The W-band EPR spectra of the PTM radicals were recorded, revealing a significant axial ganisotropy. Forbidden EPR transitions associated with flips of the ${ }^{35,37} \mathrm{Cl}$ nuclei were measured and gave the frequencies of $\mathrm{Cl}$ nuclear transitions. These showed significant overlap with the ${ }^{13} \mathrm{C}$ Larmor frequency indicating that the ${ }^{35,37} \mathrm{Cl} \rightarrow{ }^{13} \mathrm{C}$ heteronuclear polarization transfer by spin diffusion is likely. The measured ${ }^{35,37} \mathrm{Cl}$ nuclear frequencies were in good agreement with those calculated using the spin Hamiltonian parameters obtained by DFT. Further calculations of ${ }^{35,37} \mathrm{Cl}$ polarization for nuclei having resonance frequencies overlapping with ${ }^{13} \mathrm{C}$ Larmor frequency yielded ${ }^{13} \mathrm{C}$ DNP frequency sweeps that fitted the experimental ${ }^{13} \mathrm{C}$ DNP frequency sweeps better than a model based on direct ${ }^{13} \mathrm{C}$. All the reported results provide supporting evidence for the heteronuclear assisted DNP mechanism which overcome the diffusion barrier existing in the homonuclear case.

Supporting information available. Figures showing: the calculations of the ${ }^{13} \mathrm{C}$ DNP sweeps from the experimental ED-EPR spectrum, $\mathrm{T}_{1 \mathrm{e}}$ saturation recovery data, ELDOR-detected NMR spectra of six meta carboxylic acid (-COOH) analogue of radical 2 and of OX63, calculated ELDOR detected NMR spectra for spectrometer frequencies of 95,140 and $270 \mathrm{GHz}$, details on the saturation limit $(\mathrm{S} \rightarrow \infty)$ nuclear polarization enhancements of the ortho ${ }^{35} \mathrm{Cl}$ substituent of radical $\mathbf{2}$ calculated with and without separation into positive and negative polarizations, absolute values of the calculated ${ }^{13} \mathrm{C}$ DNP frequency sweeps of radicals $\mathbf{1}$ and $\mathbf{2}$, and the effect of variations of the $\mathrm{Cl}$ hyperfine and nuclear quadrupole parameters of the $13 \mathrm{C}$ DNP sweeps.

\section{Acknowledgments}

We thank Shimon Vega for valuable discussions. The research leading to these results has received funding from the DGI (Spain) under grants EMOCIONa (CTQ2006-06333), POMAs (CTQ2010-19501), and BIO2010-15683, the Networking Research Center on Bioengineering, 
Biomaterials and Nanomedicine (CIBER-BBN) promoted by ISCIII, Spain, Generalitat de Catalunya (grants 2009SGR00516 and 2009SGR1352), and the 7FP BioNMR (contract 261863). DB acknowledges Dean of Science fellowship provided by FGS, Weizmann Institute of Science. VM acknowledges the MICINN, Spain, for a Juan de la Cierva post doctoral contract. Part of the work was also supported by the German-Israeli Project Cooperation of the DFG through a special allotment by the Ministry of Education and Research (BMBF) of the Federal republic of Germany and this research was made possible in part by the historic generosity of the Harold Perlman Family. D. G. holds the Erich Klieger Professorial Chair in Chemical Physics.

\section{References}

1 A. Abragam and M. Goldman, Rep. Prog. Phys. 1978, 41, 395-467.

2 J. H. Ardenkjaer-Larsen, B. Fridlund, A. Gram, G. Hansson, L. Hansson, M. H. Lerche, R. Servin, M. Thaning and K. Golman, P. Natl. Acad. Sci. USA, 2003, 100, 10158-10163.

3 D. A. Hall, D. C. Maus, G. J. Gerfen, S. J. Inati, L. R. Becerra, F. W. Dahlquist and R. G. Griffin, Science, 1997, 276, 930-932.

4 V. Denysenkov, M. J. Prandolini, M. Gafurov, D. Sezer, B. Endeward and T. F. Prisner, Phys. Chem. Chem. Phys., 2010, 12, 5786-5790.

$5 \quad$ A. W. Overhauser, Phys. Rev., 1953, 92, 411-415.

6 A. Abragam and M. Goldman, Nuclear Magnetism: Order and Disorder, Oxford University Press, USA, 1982.

7 C. F. Hwang and D. A. Hill, Phys. Rev. Lett., 1967, 18, 110-\&.

8 M. Goldman, Spin Temperature and Nuclear Magnetic Resonance in Solids, Oxford University Press, USA, 1970.

9 T. Maly, G. T. Debelouchina, V. S. Bajaj, K. N. Hu, C. G. Joo, M. L. Mak-Jurkauskas, J. R. Sirigiri, P. C. A. van der Wel, J. Herzfeld, R. J. Temkin and R. G. Griffin, J. Chem. Phys., 2008, 128, 052211-052219.

10 F. A. Gallagher, M. I. Kettunen, S. E. Day, D. E. Hu, J. H. Ardenkjaer-Larsen, R. in't Zandt, P. R. Jensen, M. Karlsson, K. Golman, M. H. Lerche and K. M. Brindle, Nature, 2008, 453, 940U973.

11 M. E. Merritt, C. Harrison, C. Storey, F. M. Jeffrey, A. D. Sherry and C. R. Malloy, P. Natl. Acad. Sci. USA, 2007, 104, 19773-19777.

12 A. H. Emwas, M. Saunders, C. Ludwig and U. L. Gunther, Appl. Magn. Reson., 2008, 34, 483-494.

13 M. G. Saunders, C. Ludwig and U. L. Gunther, J. Am. Chem. Soc., 2008, 130, 6914-6915.

14 J. C. Paniagua, V. Mugnaini, C. Gabellieri, M. Feliz, N. Roques, J. Veciana and M. Pons, Phys. Chem. Chem. Phys., 2010, 12, 5824-5829.

15 C. Gabellieri, V. Mugnaini, J. C. Paniagua, N. Roques, M. Oliveros, M. Feliz, J. Veciana and M. Pons, Angew. Chem. Int. Edi.t, 2010, 49, 3360-3362.

16 P. Schosseler, T. Wacker and A. Schweiger, Chem. Phys. Let.t, 1994, 224, 319-324. 
17 D. Maspoch, N. Domingo, D. Ruiz-Molina, K. Wurst, G. Vaughan, J. Tejada, C. Rovira and J. Veciana, Angew. Chem. Int. Edit., 2004, 43, 1828-1832.

18 N. Roques, D. Maspoch, K. Wurst, D. Ruiz-Molina, C. Rovira and J. Veciana, Chem.-Eur. J., 2006, 12, 9238-9253.

19 D. Goldfarb, Y. Lipkin, A. Potapov, Y. Gorodetsky, B. Epel, A. M. Raitsimring, M. Radoul and I. Kaminker, J. Magn. Reson., 2008, 194, 8-15.

20 F. Neese, ORCA version 2.7 rev. 0, Bonn, 2009.

21 A. D. Becke, J. Chem. Phys., 1993, 98, 5648-5652.

22 C. T. Lee, W. T. Yang and R. G. Parr, Phys Rev B, 1988, 37, 785-789.

23 W. Kutzelnigg, U. Fleischer and M. Schindler, Ab Initio Calculation and Interpretation of NMR Chemical Shifts and Magnetic Susceptibilities, , Springer-Verlag, 1990.

24 F. Neese, J Chem Phys, 2007, 127, 164112-164119.

25 P. J. Bray and R. G. Barnes, J. Chem. Phys., 1957, 27, 551-560.

26 R. J. Lynch, T. C. Waddington, T. A. Oshea and J. A. S. Smith, J. Chem. Soc. Farad. Trans. 2, 1976, 72, 1980-1990.

27 E. Grech, J. Kalenik and L. Sobczyk, J. Chem. Soc. Farad. Trans. 1, 1985, 81, 311-319.

28 D. L. Bryce, R. P. Chapman and C. M. Widdifield, Prog. Nucl. Mag. Re.s Sp, 2009, 55, 215-

237.

29 E. A. C. Lucken, Nuclear Quadrupole Coupling Constants, Academic Press Inc.,U.S., 1969.

30 S. Sinnecker, A. Rajendran, A. Klamt, M. Diedenhofen and F. Neese, J. Phys. Chem. A, 2006, 110, 2235-2245.

31 S. Stoll and A. Schweiger, J. Magn. Reson., 2006, 178, 42-55.

32 S. K. Zaremba, Ann. Mat. Pura. Appl., 1966, 73, 293-317.

33 H. Conroy, J. Chem. Phys. 1967, 47, 5307-5318.

34 V. B. Cheng, H. H. Suzukawa and Wolfsber.M, J. Chem. Phys., 1973, 59, 3992-3999.

35 B. D. Armstrong, D. T. Edwards, R. J. Wylde, S. A. Walker and S. Han, Phy. Chem. Chem. Phys., 12, 5920-5926.

36 J. H. Ardenkjaer-Larsen, S. Macholl and H. Johannesson, Appl. Magn. Reson., 2008, 34, 509-522.

37 G. H. Rist and J. S. Hyde, J. Chem. Phys., 1970, 52, 4633-4643.

38 P. Pyykko, Molecular Physics, 2001, 99, 1617-1629.

39 V. Nagarajan, Y. Hovav, A. Feintuch, S. Vega and D. Goldfarb, J. Chem. Phys., 2010, 132, 214504-214513.

40 Y. Hovav, A. Feintuch and S. Vega, J. Magn. Reson., 2010, 207, 176-189. 


\section{Figure Captions:}

Figure 1: Molecular structures of the perchlorotryphenylmethyl (PTM) radicals 1 and $\mathbf{2}$ and the commercially available trityl radical, OX63.

Figure 2: ED-EPR spectra of the PTM radicals 1 and $\mathbf{2}$. The dotted line represents the spectrum of OX63. The magnetic fields at which ELDOR detected NMR spectra were recorded are marked on the spectrum of radical 1.

Figure 3: Comparison of the ${ }^{13} \mathrm{C}$ DNP frequency sweeps computed from the ED-EPR spectra (dashed line) and ${ }^{35,37} \mathrm{Cl}$ nuclear polarization enhancement (solid line) compared with the respective experimental DNP frequency sweep recorded at $1.5 \mathrm{~K}$ (taken form ref 15). The comparison was made after normalization of the data and a suitable shift of the frequency axis of the calculated curves.

Figure 4: (a) Pulse sequence used in the ELDOR measurements in the present work. The observation pulses were always applied at $94.9 \mathrm{GHz}$. (b) A schematic energy level diagram of a $S=1 / 2, I=3 / 2$ system with a quadrupolar coupling $\left(e^{2} Q|q| /(4 \mid(2 I-1) h)\right.$ on the order of the nuclear Larmor frequency and the hyperfine coupling. The thickness of the energy levels represents their populations, allowed EPR transitions are indicated in green and forbidden EPR transitions in red. On the right, the saturation of the 3-5 forbidden EPR transition is highlight along with the six nuclear transitions associated with level 3 (in the $M_{s}=1 / 2$ manifold) and level 5 (in the $M_{s}=-$ $1 / 2$ manifold).

Figure 5: Top: ELDOR detected NMR spectra of radicals 1 and $\mathbf{2}$ recorded at different magnetic field positions noted on the figure in $\mathrm{mT}$ units. The ${ }^{13} \mathrm{C}$ Larmor frequency is marked as a vertical dashed line in red. Bottom: The corresponding ${ }^{35,37} \mathrm{Cl}$ calculated ENDOR spectra. See text for details. The vertical dotted lines in black mark the positions of the three main peaks in the calculated spectra.

Figure 6: ELDOR measurements for both radicals 1 and $\mathbf{2}$ carried out at $g_{\perp}$ with the HTA frequency set to the ${ }^{13} \mathrm{C}$ Larmor frequency. The mw irradiation amplitude, $\omega_{1}$, is noted on the figure. 
Figure 7: Calculated DNP frequency sweeps for ${ }^{13} \mathrm{C}$ polarization mediated by the ortho ${ }^{35} \mathrm{Cl}$ of radical 1 as a function of the saturation factor $S$ (left panel) and the same plots in absolute scale (right panel). The dashed lines mark the zero intensity.

Figure 8: Calculated ${ }^{13} \mathrm{C}$ DNP frequency sweeps in the saturation limit $(S \rightarrow \infty)$. The calculations have been performed assuming that polarization takes place via a single chlorine atom: ortho (a) and meta (b) ${ }^{35} \mathrm{Cl}$ of radical 1 and ortho (c) and para (d) ${ }^{35} \mathrm{Cl}$ of radical 2. Different traces in each panel correspond to $\Delta=0.5 \mathrm{MHz}$ (black) and $\Delta=1 \mathrm{MHz}$ (red). The dashed lines mark the zero intensity. 
Figure 1
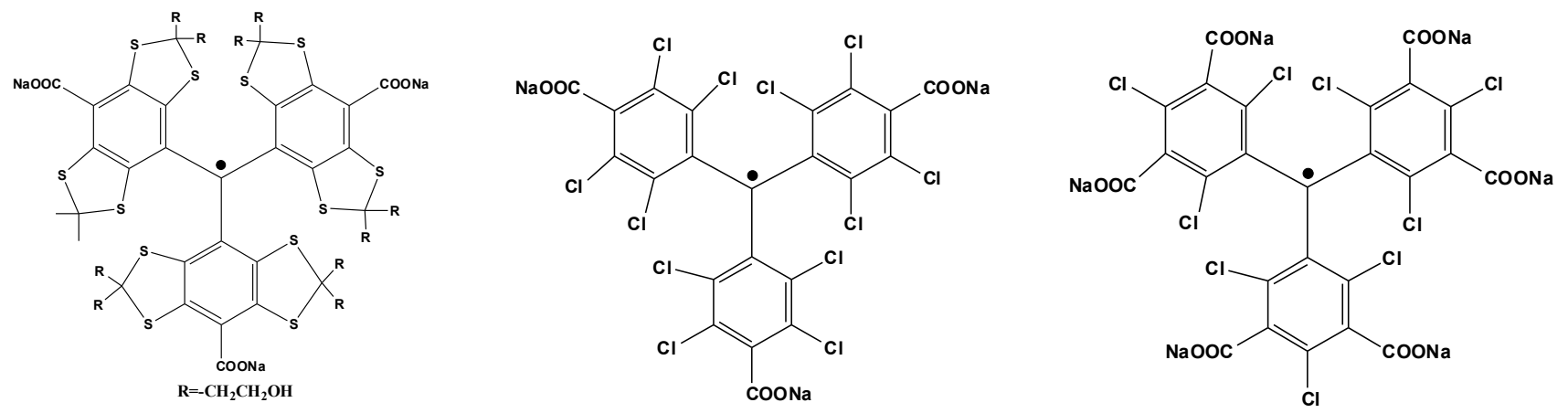
Figure 2

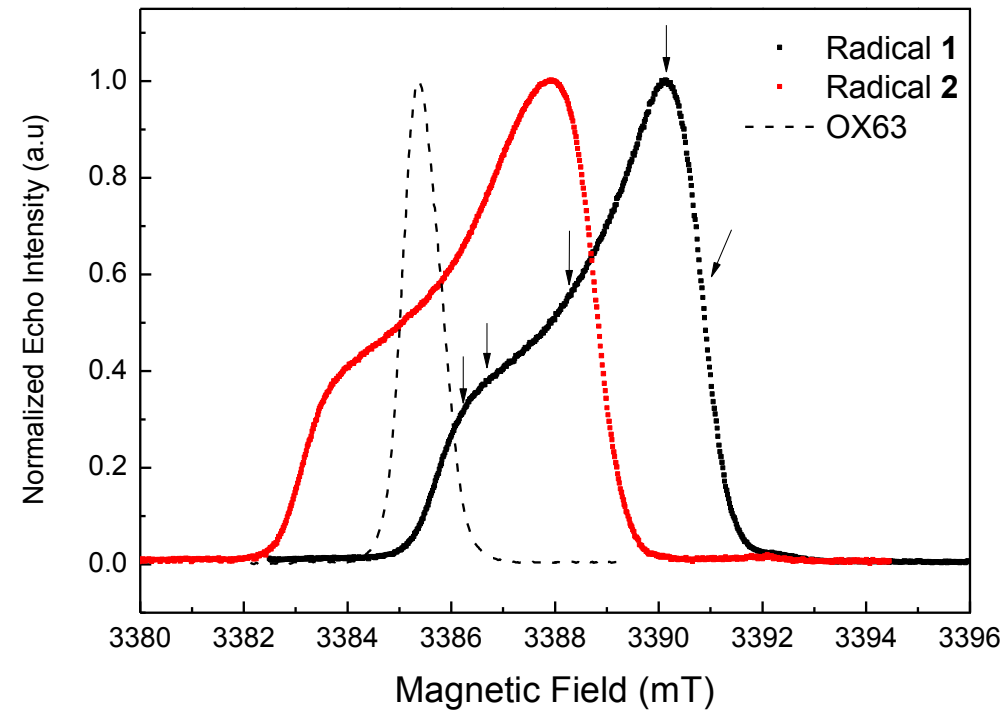


Figure 3

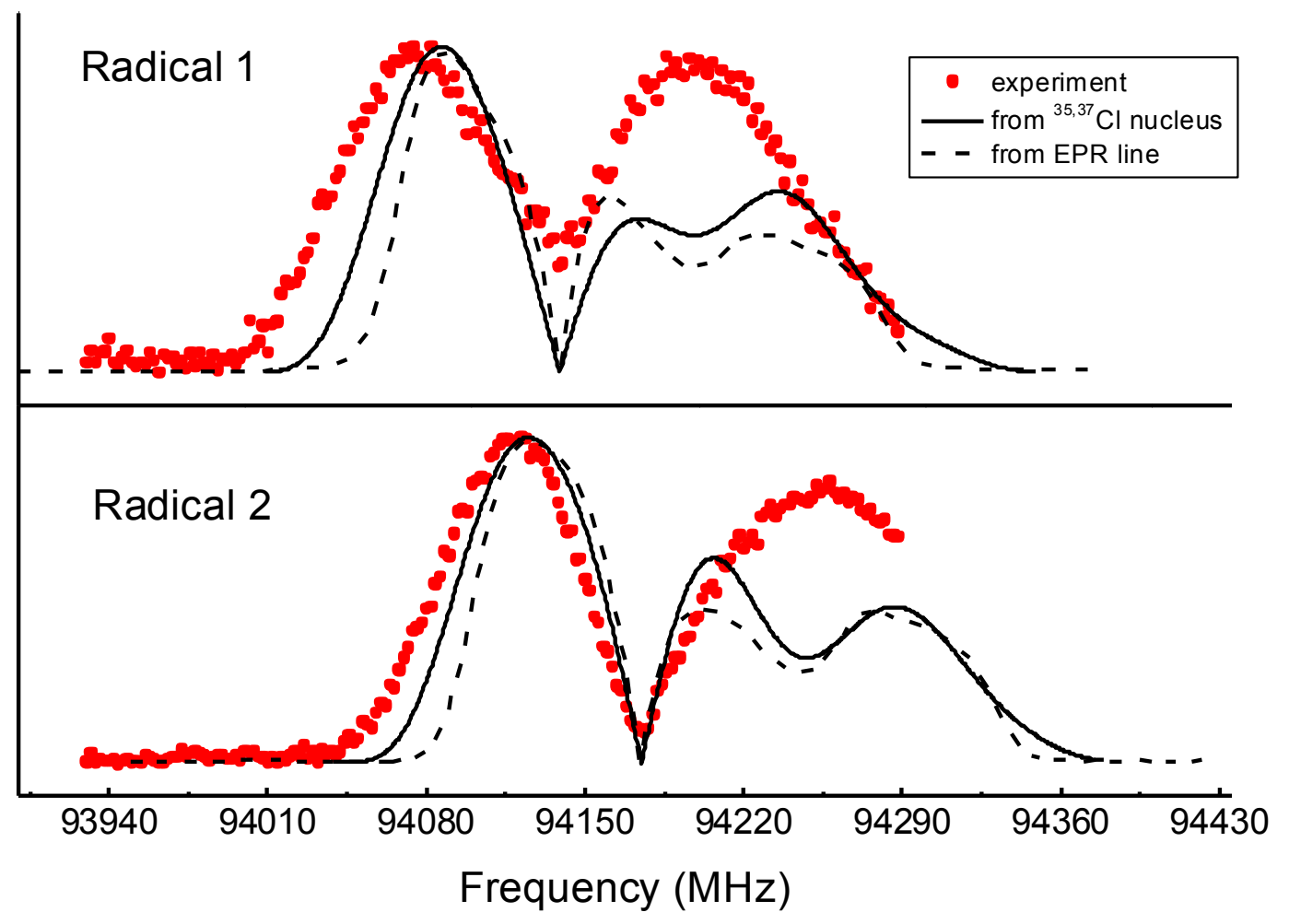


Figure 4

(a)

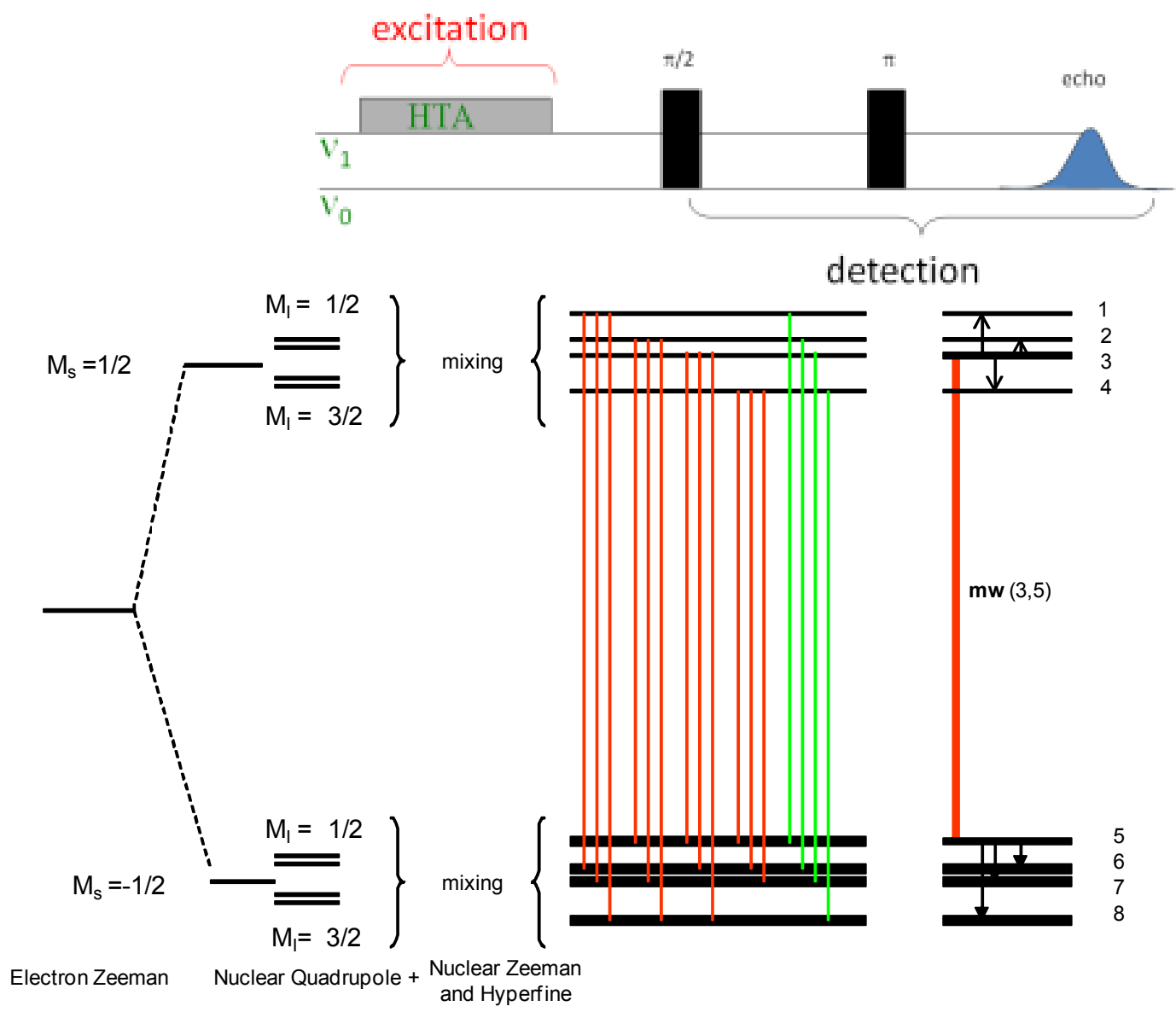

(b) 
Figure 5
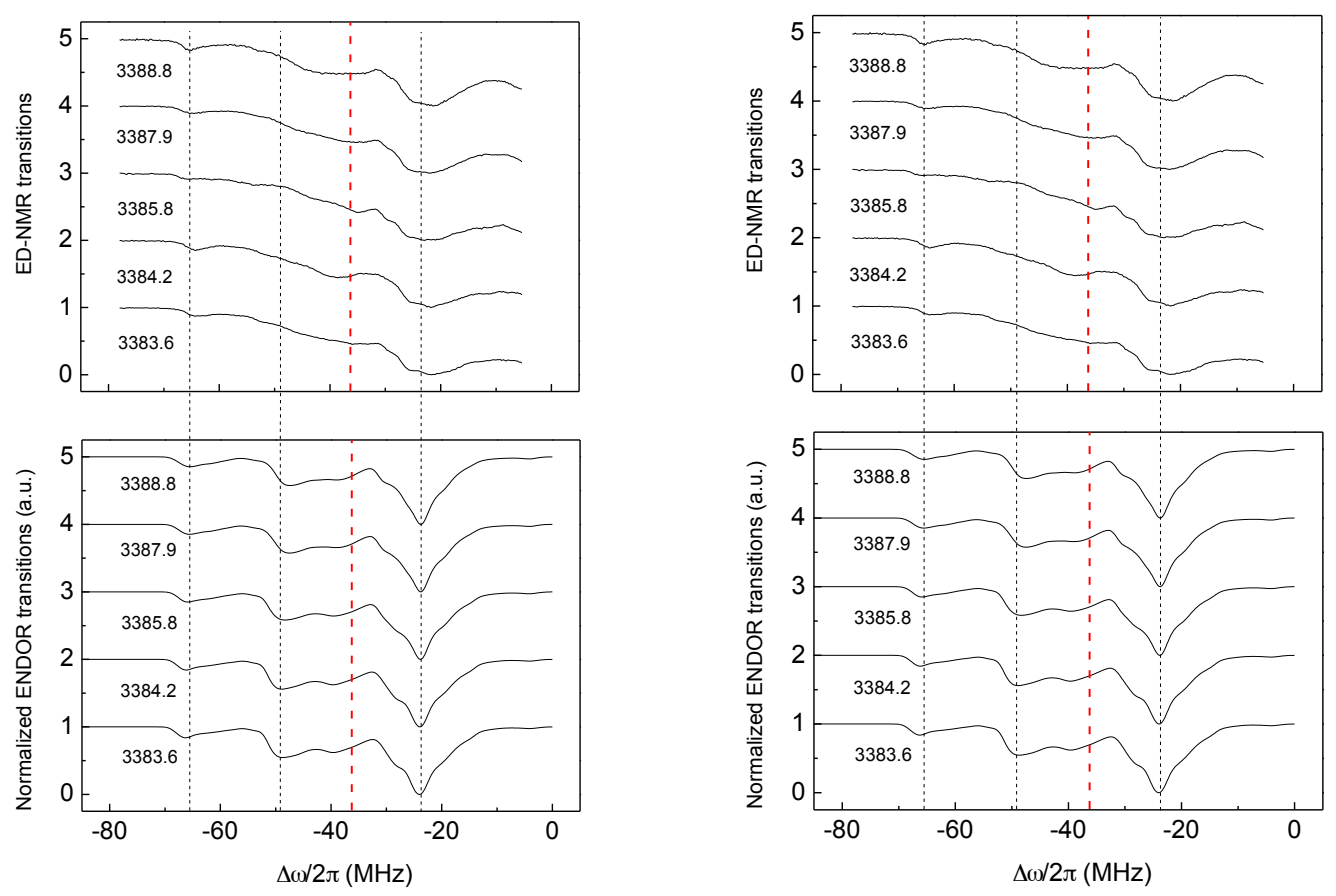
(a)

(b)

Figure 6

(c)
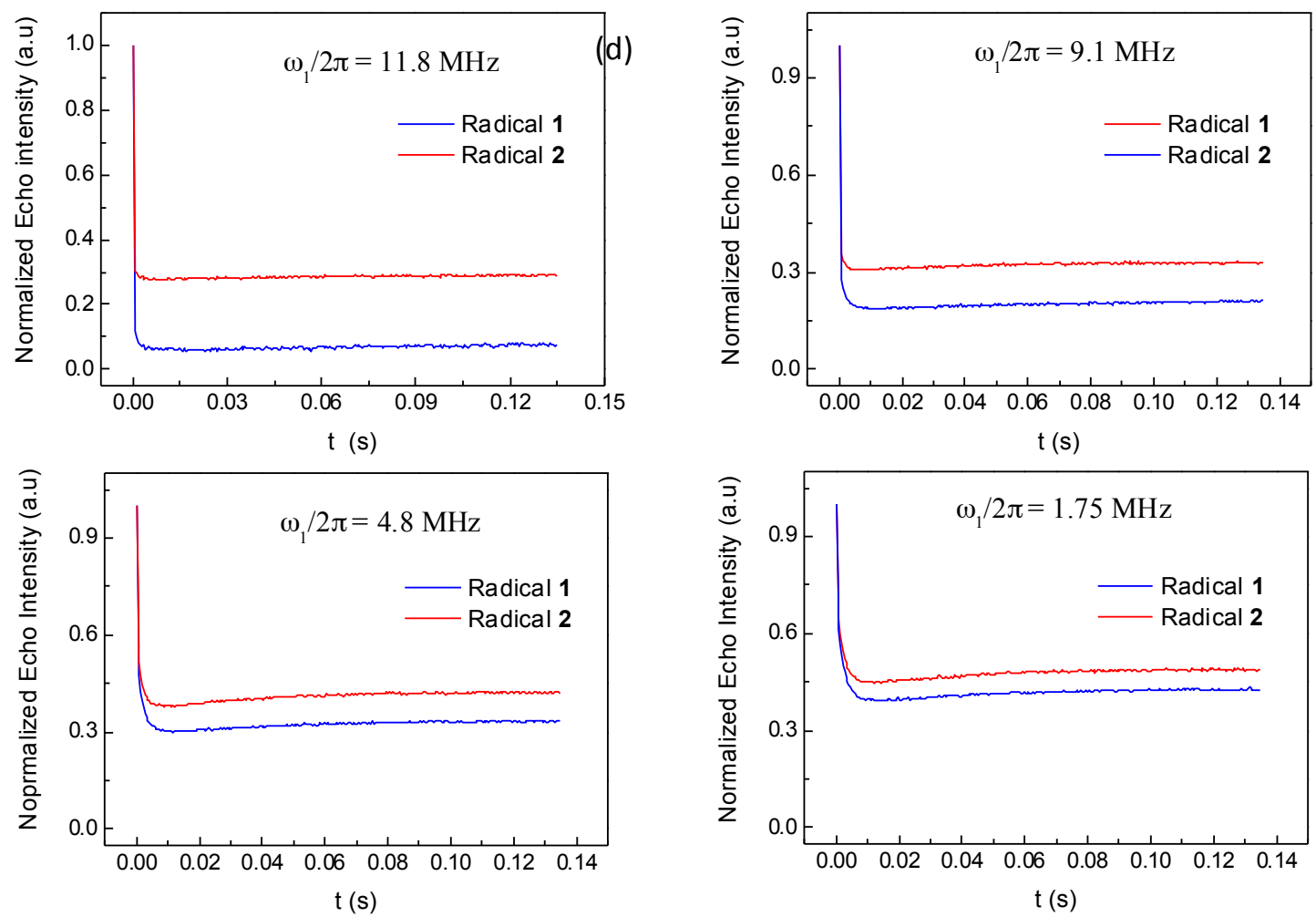
Figure 7

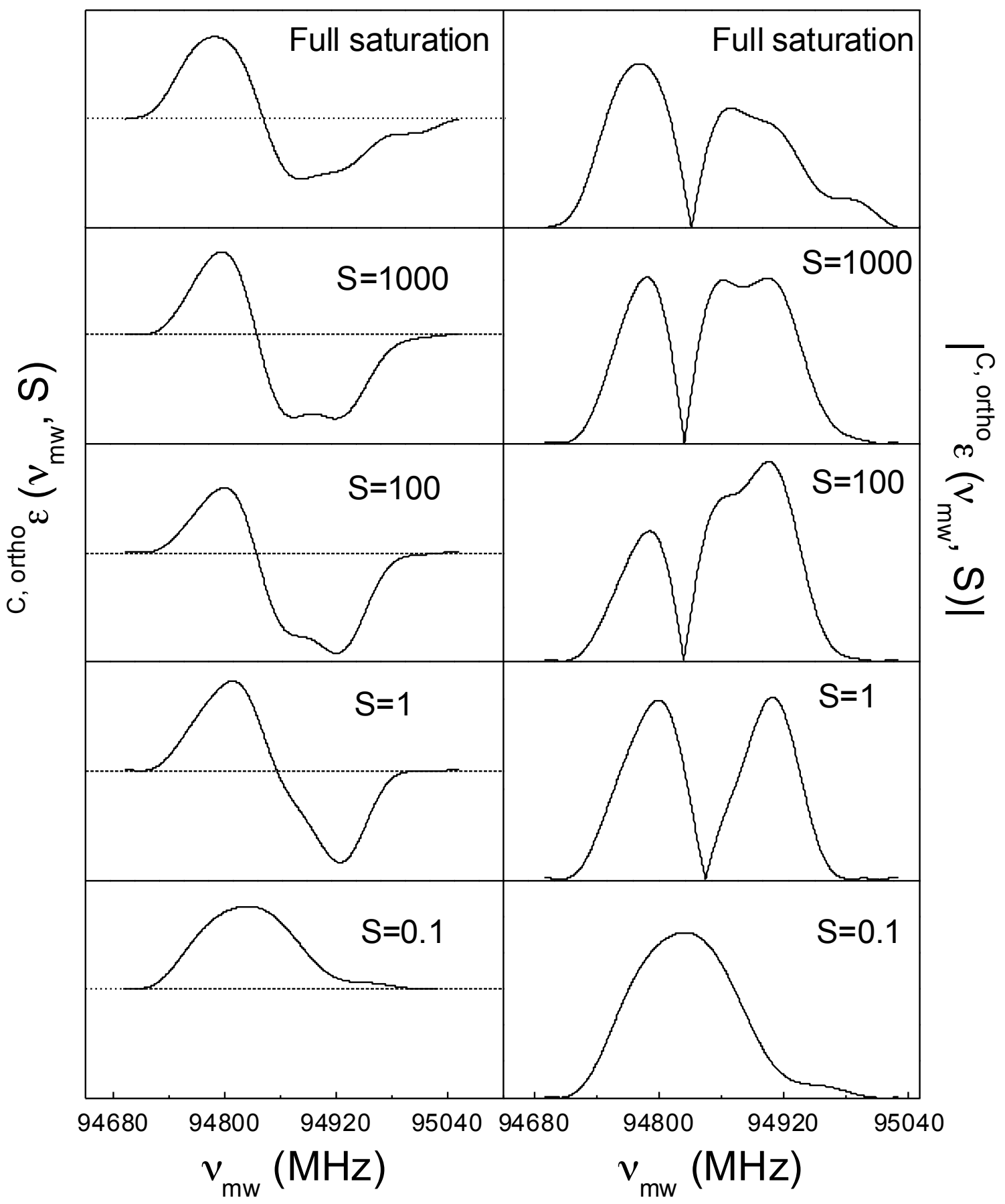


Figure 8
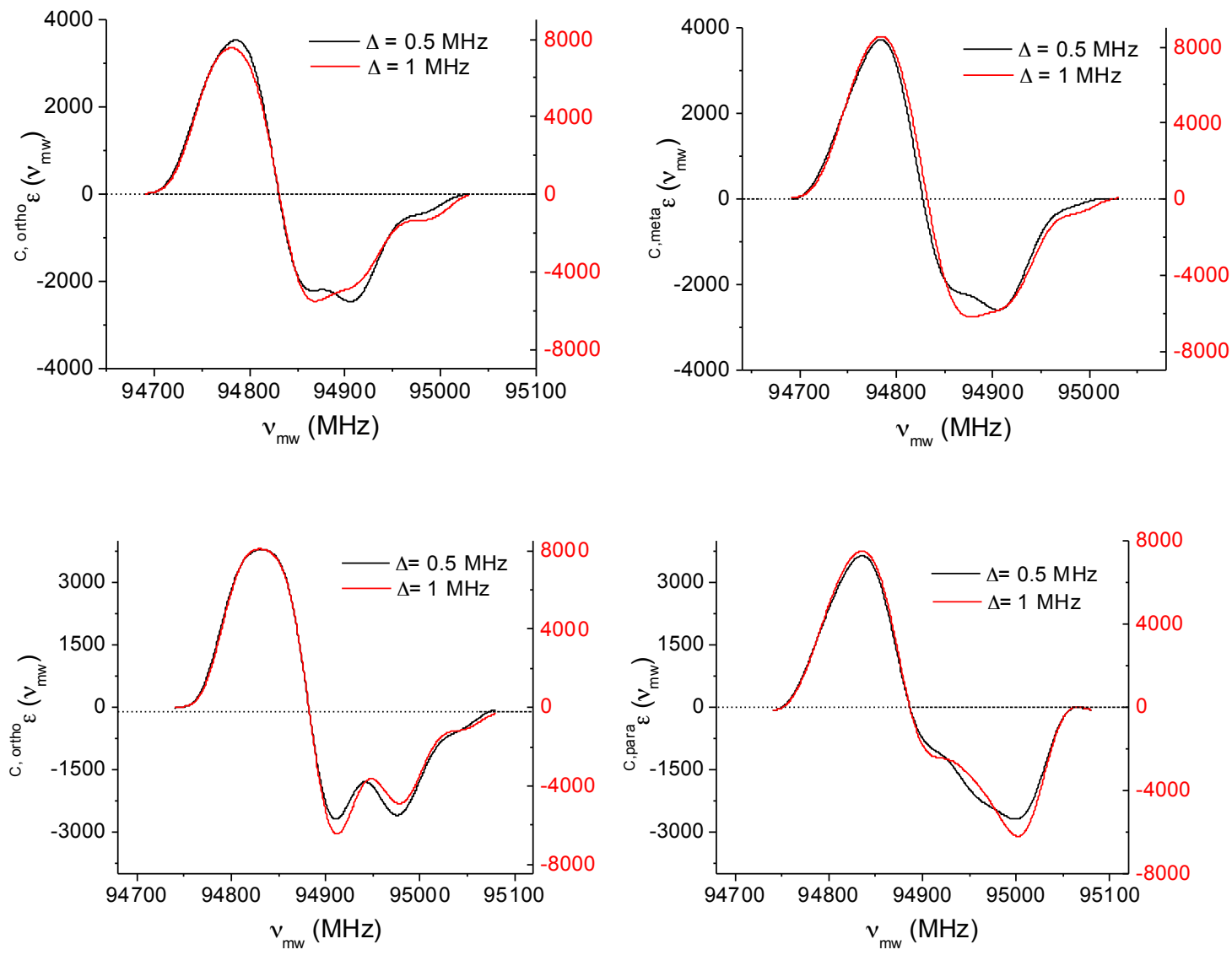
Graphical abstract:
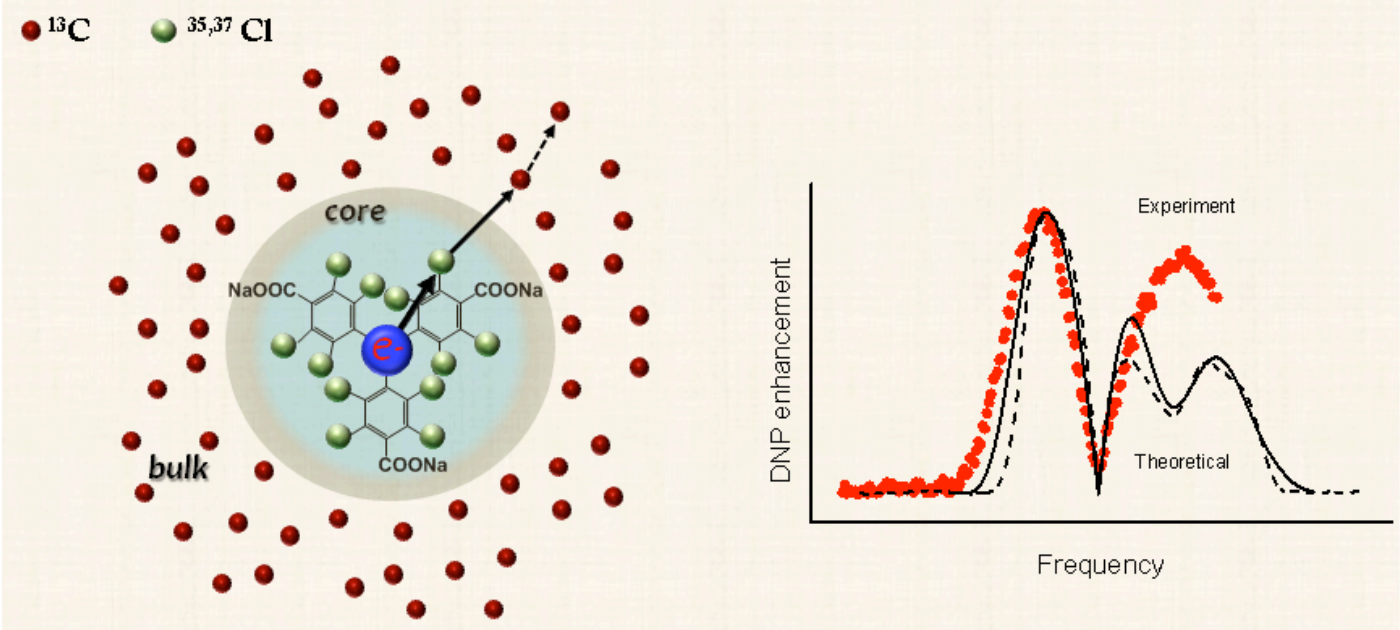

Heteronuclear assisted polarization transfer

${ }^{35,37} \mathrm{Cl}-{ }^{13} \mathrm{C}$ polarization transfer can contribute to the efficiency of polychlorotriphenylmethyl (PTM) radical as DNP polarizer. 